\title{
ARM/GCSS Single Column Model (SCM) Intercomparison Procedures for Case 3: Summer 1997 SCM IOP
}

R.T. Cerderwall, S.K. Krueger, S.C. Xie and J.J. Yio

\section{December 14, 2000}




\section{DISCLAIMER}

This document was prepared as an account of work sponsored by an agency of the United States Government. Neither the United States Government nor the University of California nor any of their employees, makes any warranty, express or implied, or assumes any legal liability or responsibility for the accuracy, completeness, or usefulness of any information, apparatus, product, or process disclosed, or represents that its use would not infringe privately owned rights. Reference herein to any specific commercial product, process, or service by trade name, trademark, manufacturer, or otherwise, does not necessarily constitute or imply its endorsement, recommendation, or favoring by the United States Government or the University of California. The views and opinions of authors expressed herein do not necessarily state or reflect those of the United States Government or the University of California, and shall not be used for advertising or product endorsement purposes.

Work performed under the auspices of the U. S. Department of Energy by the University of California Lawrence Livermore National Laboratory under Contract W-7405-Eng-48.

This report has been reproduced directly from the best available copy.

Available to DOE and DOE contractors from the

Office of Scientific and Technical Information

P.O. Box 62, Oak Ridge, TN 37831

Prices available from (423) 576-8401

http://apollo.osti.gov/bridge/

Available to the public from the National Technical Information Service

U.S. Department of Commerce 5285 Port Royal Rd., Springfield, VA 22161 http://www.ntis.gov/

OR

Lawrence Livermore National Laboratory Technical Information Department's Digital Library http://www.llnl.gov/tid/Library.html 


\title{
ARM/GCSS Single Column Model (SCM) Intercomparison Procedures for Case 3: Summer 1997 SCM IOP
}

\author{
Richard T. Cederwall, ${ }^{1}$ Steven K. Krueger, ${ }^{2}$ Shaocheng C. Xie, ${ }^{1}$ \\ and J. John Yio ${ }^{1}$
}

Go to the web (http://dev.www.arm.gov/docs/scm/scmic3) for information about the SCM Intercomparison. There you can find this documentation, as well as access to data files and associated plots. PostScript and pdf files of this documentation are available also via anonymous ftp from dev.ec.arm.gov in directory outgoing/scm_intercomp/case3_9707. See FTP procedures in section 2.3 below for further instructions.

\section{Introduction}

\subsection{ARM Objectives}

The Atmospheric Radiation Measurement (ARM) Program is a multi-laboratory, interagency program that was created in 1989 with funding from the U.S. Department of Energy (DOE). The ARM Program is part of DOE's effort to resolve scientific uncertainties about global climate change with a specific focus on improving the performance of general circulation models (GCMs) used for climate research and prediction. These improved models will help scientists better understand the influences of human activities on the earth's climate.

The objectives of the ARM Program are:

(1) to relate observed atmospheric radiation to the temperature and composition of the atmosphere, especially water vapor and clouds, across a wide range of climatologically relevant possibilities, and

(2) to develop and test improved parameterizations of radiation and radiative interactions with water vapor, aerosols, and clouds, for use in GCMs.

\subsection{SCM Working Group Objectives}

The Single-Column Model Working Group (SCM WG) is focused on the second objective of ARM. SCMs provide an economical means for testing parameterizations of physical

\footnotetext{
${ }^{1}$ Lawrence Livermore National Laboratory

${ }^{2}$ University of Utah
} 
processes active within a column of cells in a GCM. The SCM Working Group seeks to identify the data requirements for the ARM SCM research, and to facilitate scientific advances in this area by promoting collaborations among ARM Science Team members through common activities and workshops. The SCM Intercomparison is the most recent SCM research activity of the SCM Working Group. More information about the current activities and future direction of the ARM SCM WG can be found on the web (http://www.arm.gov/docs/research/scm.html), click on the link to the white paper Single Column Modeling...the Next 5 Years.

\subsection{GEWEX Cloud System Study}

The objectives of the GEWEX Cloud System Study (GCSS) are to:

(1) develop the scientific basis for the parameterization of cloud processes,

(2) highlight key issues and encourage other relevant programs to address them, and

(3) promote the evaluation and intercomparision of parameterization schemes for cloud processes.

There are a variety of cloud processes that affect the large-scale behavior of the climate system, but occur on scales too small to be represented explicity in global numerical models used for climate and weather prediction. Scientists develop numerical representations or parameterizations to represent the behavior of these processes. It is generally recognized that inadequate parameterization of clouds is one of the greatest sources of uncertainty in the prediction of weather and climate. To develop better parameterizations, GCSS efforts are organized into five working groups focused on improving our understanding of the physical processes at work within the following types of cloud systems:

1. Boundary layer

2. Cirrus

3. Extra tropical layer

4. Precipitating convective

5. Polar

\subsection{GCSS Working Group 4: Precipitating Convective Cloud Systems}

The goal of GCSS Working Group 4 (WG 4) is to improve the parameterization of precipitating convective cloud systems in global climate models (GCMs) and numerical weather prediction models through an improved physical understanding of cloud system processes. 
The main tool of GCSS WG 4 is the cloud-resolving model (CRM), also called a cloud system model (CSM), which is a numerical model that resolves cloud-scale (and mesoscale) circulations in either two or three spatial dimensions. In contrast, a GCM cannot resolve the individual convective cells or even the accompanying mesoscale circulations. Therefore, in a GCM the collective effects of these sub-grid scale processes must be parameterized. A CRM is able to determine these collective effects directly, to the extent that its representation of grid-scale dynamics and the parameterizations of its own sub-grid processes are accurate.

More information about the current and planned activities of GCSS WG4 can be found on the web (http://www.met.utah.edu/skrueger/gcss/wg4.html).

\subsection{SCM and CRM Intercomparison}

The SCM methodologies developed in the ARM Program have matured to the point where an intercomparison will help identify the strengths and weaknesses of the various approaches. In addition, the cloud system models have been compared successfully in previous intercomparison studies for tropical conditions. The ARM Southern Great Plains (SGP) Site offers an opportunity to test CRMs in continental, mid-latitude conditions. Note: The procedures for the CRM Intercomparison are contained in a separate document available on the Case 3 Intercomparison and GCSS WG4 web pages.

\subsubsection{Objectives}

In this intercomparison study, we seek to provide a common set of forcing data, and supporting data, for running the SCMs and CRMs. In this way, the differences in results will be due to differences in the SCM and CRM parameterizations, not the input data sets. We seek to evaluate the results from the participating models to address several issues, including the optimal method for obtaining representative results from SCMs via ensemble and multiple-run schemes, the isolation of cloud parameterization effects by prescribing the radiative heating, effects of prescibed surface forcing, and the performance of the SCMs and CRMs themselves.

\subsubsection{Description}

The intercomparison is based on observations collected during the SCM IOP that took place from 18 June to 18 July 1997 at the ARM SGP Site. The comparison runs will cover 18 June (2330 UTC) to 17 July (2330 UTC). This IOP was chosen for the ARM/GCSS case study since it was convectively driven, and the period contained a wide range of summertime weather conditions. 


\section{Numerical Experiment Protocol}

\subsection{Description for SCM Experiments}

\subsubsection{Initial conditions}

The initial profiles are based on the observed SGP CART areal averages of temperature, water vapor mixing ratio, and horizontal wind velocity. These are provided as functions of pressure at DP mb intervals from BOT to TOP mb. For the values derived by Zhang's variational analysis, $\mathrm{DP}=50 \mathrm{mb}, \mathrm{BOT}=965 \mathrm{mb}$, and $\mathrm{TOT}=115 \mathrm{mb}$. The values at BOT mb represent those of the layer that starts at the surface. The values provided must be interpolated to each model's vertical grid levels.

To obtain values for model levels below BOT mb by interpolation, assume that $\bar{\theta}, \bar{q}$,

$\bar{u}$, and $\bar{v}$ at the surface are equal to their values at BOT mb, and that $(\partial \bar{T} / \partial t)_{\text {L.S. }}$, and $(\partial \bar{q} / \partial t)_{\text {L.S. }}$ are equal to zero at the surface. $\bar{\omega}$ is defined by the surface pressure tendency $\partial p_{s} / \partial t$; we have neglected $v_{s} \cdot \nabla p_{s}$.

To obtain values for model levels above TOP mb by interpolation, the simplest approach is to assume that $\bar{T}, \bar{q}, \bar{u}$, and $\bar{v}$ at $20 \mathrm{~km}$ are equal to their values at TOP mb, and that $\bar{\omega},(\partial \bar{T} / \partial t)_{\text {L.S. }}$, and $(\partial \bar{q} / \partial t)_{\text {L.S. }}$ are equal to zero at $20 \mathrm{~km}$.

Radiative transfer calculations may require temperature, water vapor mixing ratio, and ozone mixing ratio profiles above $20 \mathrm{~km}$. The "standard mid-latitude summer atmosphere" profiles are available for this purpose, and can be found on the SCM Intercomparison web page under SCM Supporting Data Sets.

\subsubsection{Upper and lower boundary conditions}

The lower boundary is a land surface. For this intercomparison, values of surface sensible and latent heat flux are given as surface forcing for the models. The surface forcing terms are obtained from site-wide averages of the observed fluxes by the ARM Energy Balance Bowen Ratio (EBBR) stations. The ground surface temperature is also specified in the forcing data sets. For diagnostic purposes, other surface quantities are provided hourly, including surface pressure,soil temperature and moisture, near-surface winds, downwelling solar and IR radiation, and precipitation. The average elevation of the ARM SGP SCM domain is $360 \mathrm{~m}$.

Radiative transfer calculations require the solar constant and the solar zenith angle (or its cosine). Due to the variation of the earth-sun distance $d$ from its mean value $d_{m}$, the solar "constant" $S$ is adjusted from its mean value of $S_{m}=1365 \mathrm{~W} / \mathrm{m}^{2}$. For the period June 18 - July 17 , the average value for the ratio $\left(d_{m} / d\right)^{2}=0.9676$, so $S=S_{m}\left(d_{m} / d\right)^{2}=$ $1321 \mathrm{~W} / \mathrm{m}^{2}$.

You may use the Fortran subroutine listed below (available via the SCM Intercomparison web page, under SCM Supporting Data Sets, and also via anonymous ftp in file 
solar99.f) to calculate the cosine of the solar zenith angle. The central facility of the SGP CART is located at latitude $36^{\circ} 36^{\prime} \mathrm{N}$, longitude $97^{\circ} 29^{\prime} \mathrm{W}$, at an elevation of 315 $\mathrm{m}$ above sea level.

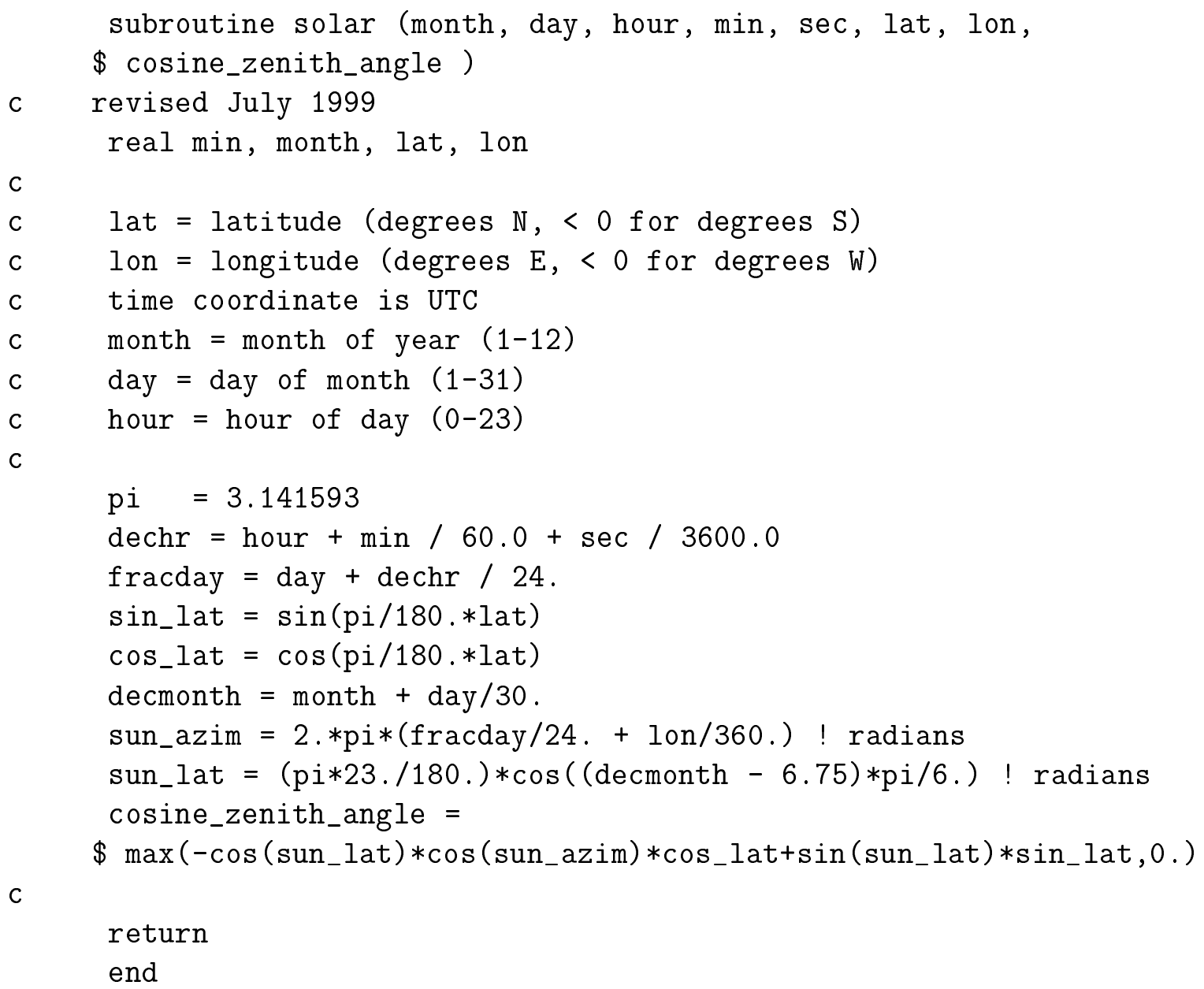

\subsubsection{Large-scale forcing}

The large-scale forcing terms in the SCM governing equations include the large-scale horizontal pressure gradient force, and the large-scale advective tendencies of horizontal momentum,temperature, and water vapor mixing ratio. The SCM horizontal grid sizes are considered to be small compared to large-scale disturbances. In this case, the largescale forcing will be approximately uniform over the model domains. For this reason, the large-scale forcing terms are defined to be functions of height and time only. The available large-scale forcing terms are based on observations averaged over the 12-sided variational analysis grid for SGP CART region (about $370 \mathrm{~km}$ across). 
Temperature and water vapor mixing ratio The large-scale advective tendencies for temperature $T$ and water vapor mixing ratio $q$ are formally defined (on isobaric surfaces) as

$$
\left(\frac{\partial \bar{T}}{\partial t}\right)_{\text {L.S. }} \equiv-\overline{\mathbf{v}} \cdot \nabla \bar{T}-\bar{\omega} \frac{\partial \bar{T}}{\partial p}+\frac{\bar{\omega}}{c_{p}} \alpha
$$

and

$$
\left(\frac{\partial \bar{q}}{\partial t}\right)_{\text {L.S. }} \equiv-\overline{\mathbf{v}} \cdot \nabla \bar{q}-\bar{\omega} \frac{\partial \bar{q}}{\partial p},
$$

where $\nabla$ is the horizontal del operator. Note that for potential temperature

$$
\left(\frac{\partial \bar{\theta}}{\partial t}\right)_{\text {L.S. }}=\left(\frac{p_{0}}{\bar{p}}\right)^{R / c_{p}}\left(\frac{\partial \bar{T}}{\partial t}\right)_{\text {L.S. }} .
$$

SCMs and CRMs are unable to calculate $\nabla \bar{T}, \nabla \bar{q}$, or $\bar{\omega}$, so these must be specified from observations. In the models, $(\partial \bar{T} / \partial t)_{\text {L.S. }}$ and $(\partial \bar{q} / \partial t)_{\text {L.S. will be calculated using one }}$ of the following three methods.

Unfortunately, observations of the advective tendencies for hydrometeors are not available. This may have a significant impact on the simulation of middle and upper tropospheric stratiform clouds.

The lateral forcing will be specified in two different ways for the intercomparison runs: total (or revealed) forcing, and total plus relaxed forcing. Each is described below.

\section{Specify the observed total advective tendency (revealed)}

In this method,

$$
\left(\frac{\partial \bar{T}}{\partial t}\right)_{\text {L.S. }}=\left(\frac{\partial \bar{T}}{\partial t}\right)_{\text {L.S.T. }}
$$

and

$$
\left(\frac{\partial \bar{q}}{\partial t}\right)_{\text {L.S. }}=\left(\frac{\partial \bar{q}}{\partial t}\right)_{\text {L.S.T. }},
$$

where $(\partial \bar{T} / \partial t)_{\text {L.S.T. }}$ and $(\partial \bar{q} / \partial t)_{\text {L.S.T. }}$ are the observed large-scale total advective tendencies for $T$ and $q$.

Specify total advective tendency plus relaxation toward observed value . In this method,

$$
\left(\frac{\partial \bar{T}}{\partial t}\right)_{\text {L.S. }}=\left(\frac{\partial \bar{T}}{\partial t}\right)_{\text {L.S.T. }}+\frac{\bar{T}-\bar{T}_{\text {model }}}{\tau_{a}}
$$

and

$$
\left(\frac{\partial \bar{q}}{\partial t}\right)_{\text {L.S. }}=\left(\frac{\partial \bar{q}}{\partial t}\right)_{\text {L.S.T. }}+\frac{\bar{q}-\bar{q}_{\text {model }}}{\tau_{a}}
$$


, where $\bar{T}, \bar{q}$, and $\tau_{a}$ are the observed large-scale temperature, mixing ratio, and advective time scale. Note, again, that potential temperature can be obtained by

$$
\bar{\theta}=\left(\frac{p_{0}}{\bar{p}}\right)^{R / c_{p}} \bar{T} .
$$

For this intercomparison, the advective time scale $\tau_{a}$ is defined as

$$
\tau_{a}=\frac{D}{2 \sqrt{u^{2}+v^{2}}}
$$

where $D$ is the distance across the SCM domain (specified here as $370 \mathrm{~km}$ ), and $u$ and $v$ are the wind components in the column from the analyses.

Profile values are given at 50-mb intervals for the SUNY variational analysis, and at 3-hour intervals. These values must be interpolated in pressure to the model's grid and in time to obtain values at each time step.

\subsubsection{Numerical simulations}

As a reference for understanding the numerical simulation periods, average precipitation is plotted in Figure 1. The simulations defined below capture convective, precipitating periods and non-precipitating or dry periods. In our case labeling, capital letters designate time periods, while numbered suffixes refer to simulation approaches. Periods are characterized as precipitating or dry. We begin with the simulation approaches that describe the forcing used and other related simulation conditions.

\section{Simulation approaches (forcing) for SCM Intercomparison:}

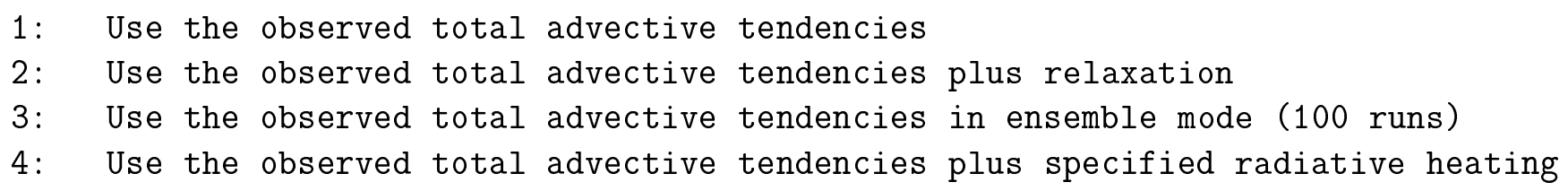

Approaches \#1, \#2 and \#4 are required, while Approach \#3 is recommended.

For Approach \#3, the perturbed initial profiles of temperature and moisture are provided via the SCM Intercomparison web page. Results for the ensemble simulations are reported as mean and standard deviations of quantities across the ensemble.

Approach \#4 is the same forcing as that used in Phase I of the CRM Intercomparison. For Approach \#4, the specified radiative heating rate profiles are given on the web page, and are used in place of the values normally obtained from the radiation model. They are obtained from diagnostics from the ECMWF model provided by Christian Jakob, and adjusted to be consistent with the variational analysis. See Section 2.3 in the CRM Intercomparison Procedures (on the web) for more details. 


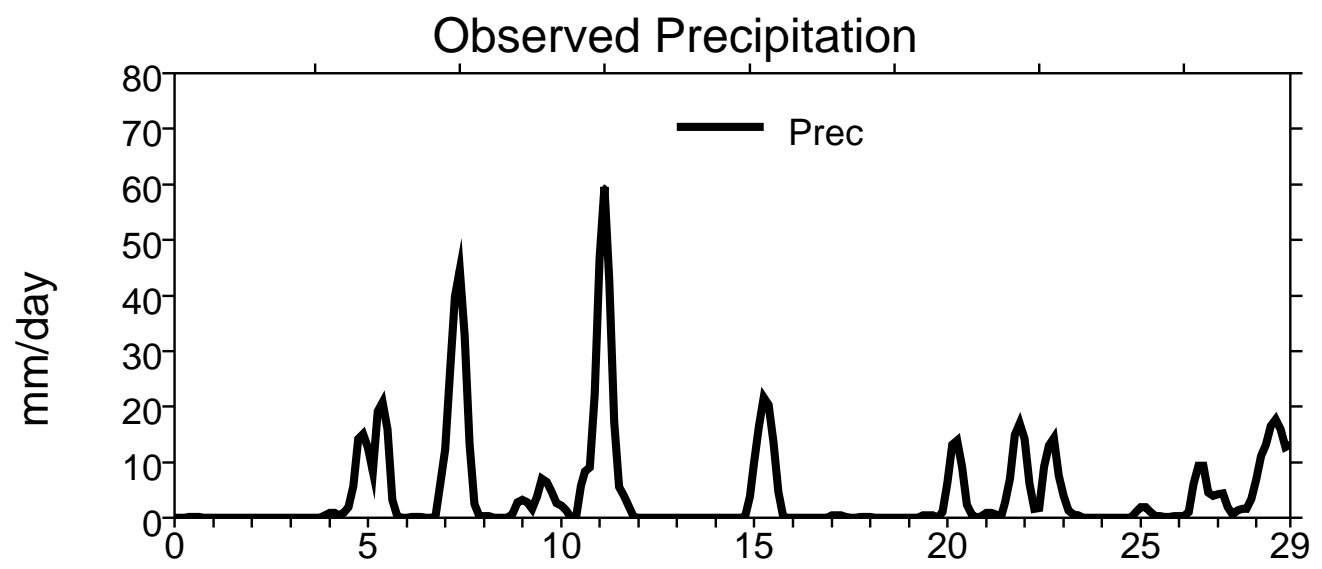

Time (Days since 2330 UTC 18 June 1997)

Figure 1: Average precipitation (mm/day) in the SCM analysis domain.

\section{Case periods in common with the CRM Intercomparison:}
A: 2330 UTC 26 June 1997 - 2330 UTC 30 June 1997
4 days precip
B: 2330 UTC 7 July 1997 - 2330 UTC 12 July 1997
5 days
precip
C: 2330 UTC 12 July 1997 - 2330 UTC 17 July 1997
5 days
precip

\section{Case periods for SCM Intercomparison only:}

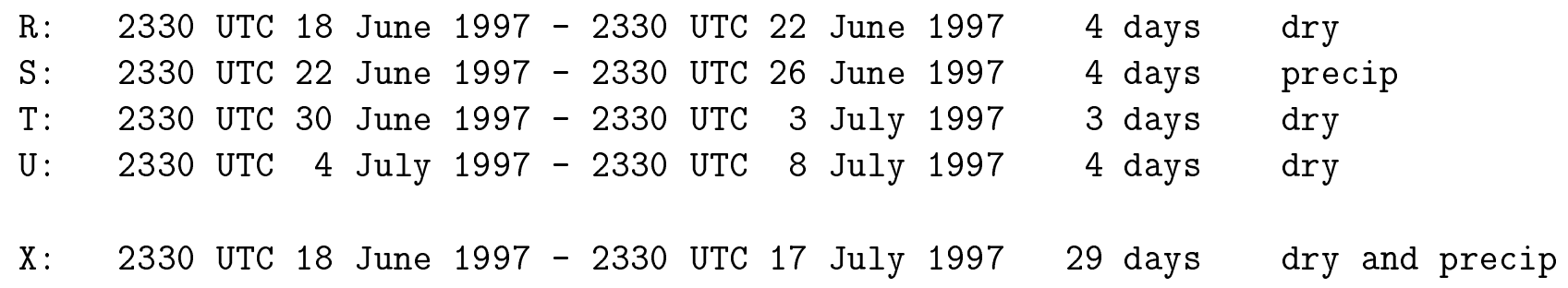

The Case periods $\mathbf{R}, \mathbf{S}, \mathbf{T}$, and $\mathbf{U}$ provide additional study periods for the SCM Intercomparison, especially for non-precipitating periods. Case period $\mathbf{X}$ is included to study SCM performance in terms of model drift over a long period. 


\subsection{Obtaining Data and Submitting Results}

\subsubsection{Accessing and reading the files}

Data files for the ARM/GCSS SCM and CSM Intercomparison will be at the ARM Experiment Center. They are accessible by FTP, as follows:

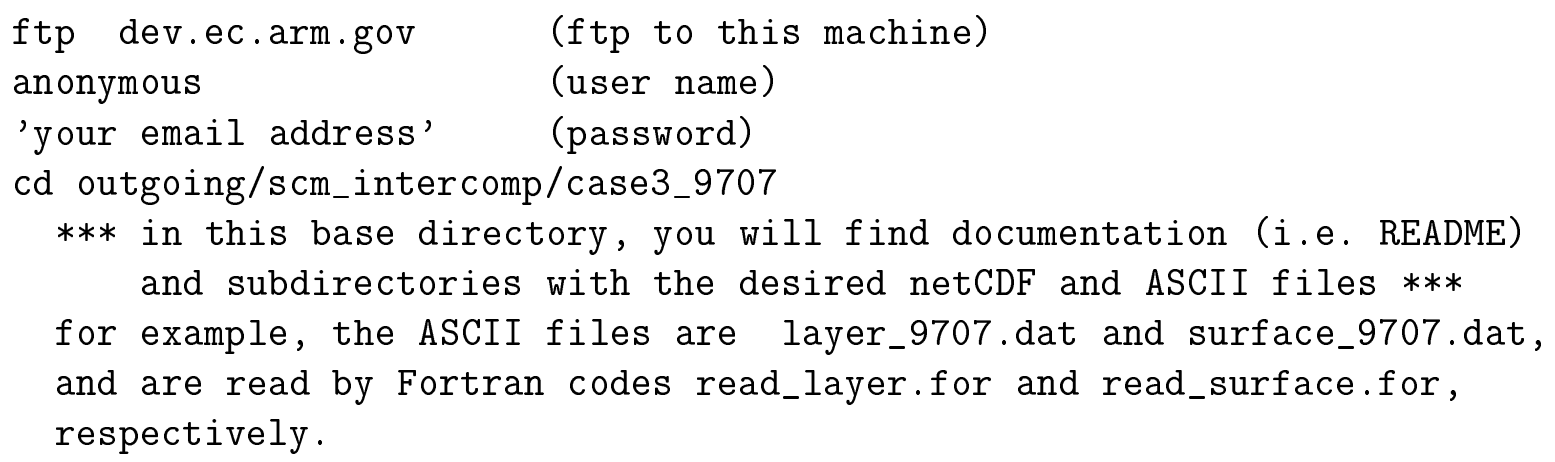

Vertical profiles of observed large-scale temperature $\left(\bar{T}_{\text {obs }}\right)$, mixing ratio $\left(\bar{q}_{\text {obs }}\right)$, and velocity components $\left(\bar{u}_{\text {obs }}, \bar{v}_{\text {obs }}\right.$, and $\left.\bar{\omega}\right)$; the observed ground surface temperature $\left(\bar{T}_{s}\right)$ and the surface pressure $\left(\bar{p}_{0}\right)$; profiles of the observed large-scale total advective tendencies of temperature and mixing ratio $\left((\partial \bar{T} / \partial t)_{\text {L.S.T. }}\right.$ and $\left.(\partial \bar{q} / \partial t)_{\text {L.S.T. }}\right)$, and horizontal and vertical advective tendencies of temperature and mixing ratio $\left((\partial \bar{T} / \partial t)_{\text {L.S.H. }}\right.$ and $\left.(\partial \bar{q} / \partial t)_{\text {L.S.H. }}\right)$ are contained here.

Values are given for temperature, instead of potential temperature, for all terms, and for dry static energy for some terms. Note: use 'Horizontal_Temp_Advec' + 'Vertical_s_Advec' for total advective temperature tendency to account for abiabatic compression term. Several temperature terms can be converted to potential temperature using the conversion factor:

$$
\left(\frac{p_{0}}{\bar{p}}\right)^{R / c_{p}}
$$

where $p_{o}$ is $1000 \mathrm{mb}$.

\subsubsection{Submitting output files}

Files containing results of your runs can be submitted via FTP to the ARM Experiment Center. Modify the FTP procedures above by accessing a different directory to put your result files:

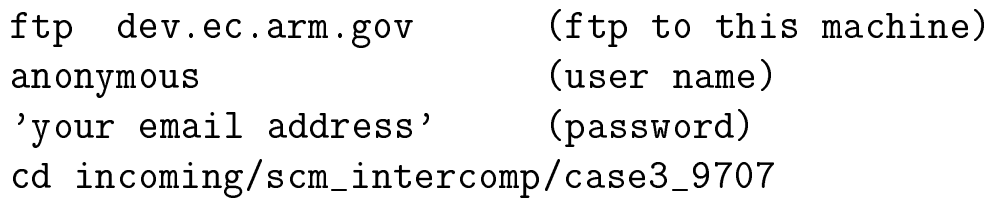


Once you are in that directory, you may create your own subdirectory using mkdir your_directory_name. For our convenience, use your last name for the directory name, unless you have another preference. Files are automatically removed from the FTP directory 14 days after your submission, so we must transfer them to other long-term directories within that time. It will help us if you send email to John Yio (yio@llnl.gov) and Ric Cederwall (rcederwall@llnl.gov) after you submit results so that we will make sure that your result files are handled in a timely manner.

We are asking participants to submit results for several simulations. To make sure that the proper format is being used, we encourage participants to submit results of at least one simulation EARLY in their efforts. Once we check that the files are in the correct format, then you can bundle up the remaining results and submit them. This saves everyone unnecessary effort in the long run.

Naming of files: Name each file as follows: simulation.item. model name. Simulation names are a combination of the Case Period and the Approach used for forcing; for example, a run for Case period A using simulation Approach 2 would be named 'a2'. Note that we are using lower case letters for the case periods. Item names include BOTH whether it is a profile ( $\mathrm{p}$ ) or time series ( $\mathrm{t}$ ), followed by a number representing the item number if a profile or the group number if a time series. The model name is up to the participant. It can include the name of the group or the modeler, i.e. CSU-SCM. Names cannot exceed 13 characters and must not include the characters '/' or '.', since we will parse the model name and use it in plots we generate for submitted results. With these conventions, the file name "a2.p2.CSU-SCM", for example, indicates simulation=a2, item $=\mathrm{p} 2$ profile (temperature), and model name $=$ CSU-SCM. 


\section{Results to Submit}

The results to be submitted will consist of large-scale quantities unless otherwise noted. Large-scale is defined as both a space and time average, indicated by an overbar. The SCM quantities already represent a space average in the horizontal direction over the SCM domain. The time average is the average over a 3-hour period, and should be based on "observations" taken frequently enough to avoid aliasing due to cloud-scale and mesoscale variability. Some quantities, such as surface rainfall, should be accumulated every time step. Unless noted otherwise, time averages should be obtained from observations taken every SCM time step (approximately 20 minutes).

The large-scale quantities to be submitted will consist of time series and profiles at 3 -hourly intervals. The time series will be largely based on quantities provided in more detail in the profiles, so the latter will be described first. For the ensemble mode, report means and standard deviations of requested quantities for the

Instructions on how to submit the files containing the quantities described below have been outlined in Section 2.3.2 above. If you have questions, you may contact John Yio via e-mail at yio@llnl.gov. We have chosen to use ASCII format for submitting results as a convenience for participants. We convert submitted data files to netCDF format for the common database. You may submit your results in netCDF format now if you prefer. Please put the profile data in a separate file from the time series (which themselves can go in three separate files).

A description of quantities to submit for the SCMs and CSMs follows in the next two subsections. After that is a subsection with additional information on file format. The bottom line is that we are using a flexible ASCII format, which is quite robost. To reduce the file sizes, suggested formats for each quantity are included in the descriptions below using Fortran notation; however, these are not mandatory. At a minimum, we need integer, fixed, or exponential formatted fields that are delimited by spaces, tabs, or end-of-lines. What is important for the profile quantities is that pressure (or height) be the fastest moving variable, followed by time. 


\subsection{Description of quantities for SCM Experiments}

Associated with each of the quantities below is a priority, in bold type and brackets $<>$. The priorities are:

$\begin{array}{ll}1 & \text { required } \\ 2 & \text { recommended } \\ 3 & \text { optional }\end{array}$

\subsubsection{Profiles}

Submit the results for each quantity listed below as a separate ASCII file in the file format described in the subsection after the quantity descriptions.

1. $<\mathbf{1}>$ Height, $z(\mathrm{~km})$ (F7.3) if on constant pressure surface, or Pressure, $p(\mathrm{mb})(\mathrm{F} 7.3)$ if on constant height surface

2. $<\mathbf{1}>$ Temperature, $\bar{T}(\mathrm{~K})(\mathrm{F} 7.2)$

3. $<\mathbf{1}>$ Water vapor mixing ratio, $\bar{q}(\mathrm{~g} / \mathrm{kg})(\mathrm{F} 7.3)$

4. $<$ 2>Relative humidity, $R$ (unitless) (F6.3): $R=\bar{q} / q^{*}(\bar{T}, \bar{p})$, where $q^{*}(T, p)$ is the saturation mixing ratio over water.

5. $<\mathbf{1}>$ Cloud water (suspended liquid water) mixing ratio, $\overline{q_{c}}(\mathrm{~g} / \mathrm{kg})(\mathrm{F} 7.4)$

6. $<\mathbf{1}>$ Cloud ice (suspended ice) mixing ratio, $\overline{q_{i}}(\mathrm{~g} / \mathrm{kg})(\mathrm{F} 7.4)$

7. $<3>$ Rain (falling liquid water) mixing ratio, $\overline{q_{r}}(\mathrm{~g} / \mathrm{kg})(\mathrm{F} 7.4)$

8. $<\mathbf{3}>$ Snow (slow-falling ice) mixing ratio, $\overline{q_{s}}(\mathrm{~g} / \mathrm{kg})(\mathrm{F} 7.4)$

9. $<\mathbf{3}>$ Graupel (fast-falling ice) mixing ratio, $\overline{q_{g}}(\mathrm{~g} / \mathrm{kg})(\mathrm{F} 7.4)$

10. $<\mathbf{1}^{*}>$ Cloud fraction, $\bar{\sigma}$ (unitless) (F6.3): At each grid point, $\sigma=1$ if $q_{c}+q_{i}>$ $0.01 q^{*}(\bar{T}, \bar{p})$; otherwise, $\sigma=0$.

11. $<\mathbf{2}>$ Horizontal wind velocity in $\mathrm{x}$-direction, $\bar{u}(\mathrm{~m} / \mathrm{s})(\mathrm{F} 7.2)$

12. $<\mathbf{2}>$ Horizontal wind velocity in y-direction, $\bar{v}(\mathrm{~m} / \mathrm{s})(\mathrm{F} 7.2)$

13. $<\mathbf{1}>$ Apparent heat source, $Q_{1 C}(\mathrm{~K} /$ day $)(\mathrm{F} 7.2)$ :

$$
Q_{1 C} \equiv Q_{1}-Q_{R}=\left[\frac{\partial \bar{T}}{\partial t}-\left(\frac{\partial \bar{T}}{\partial t}\right)_{\text {L.S. }}\right]-Q_{R}
$$

where

$$
\left(\frac{\partial \bar{T}}{\partial t}\right)_{\text {L.S. }} \equiv-\overline{\mathbf{v}} \cdot \nabla \bar{T}-\bar{\omega} \frac{\partial \bar{T}}{\partial p}
$$

is the large-scale advective tendency (or "forcing") which is specified from observations. 
14. $<\mathbf{1}>$ Apparent moisture sink, $Q_{2}(\mathrm{~K} /$ day) $(\mathrm{F} 7.2)$ :

$$
Q_{2}=-\frac{L}{c_{p}}\left[\frac{\partial \bar{q}}{\partial t}-\left(\frac{\partial \bar{q}}{\partial t}\right)_{\text {L.S. }}\right],
$$

where

$$
\left(\frac{\partial \bar{q}}{\partial t}\right)_{\text {L.S. }} \equiv-\overline{\mathbf{v}} \cdot \nabla \bar{q}-\bar{\omega} \frac{\partial \bar{q}}{\partial p}
$$

is the large-scale advective tendency (or "forcing") which is specified from observations.

15. $<3>$ Convective $Q_{1 C}, Q_{1 C}^{\mathrm{c}}\left(\mathrm{K} /\right.$ day) $(\mathrm{F} 7.2)$ : Contribution to $Q_{1 C}$ from the "convective" columns.

16. $<3>$ Stratiform $Q_{1 C}, Q_{1 C}^{\mathrm{s}}\left(\mathrm{K} /\right.$ day) (F7.2): Contribution to $Q_{1 C}$ from the "stratiform" columns.

17. $<3$ Convective $Q_{2}, Q_{2}^{\mathrm{c}}$ (K/day) (F7.2): Contribution to $Q_{2}$ from the "convective" columns.

18. $<3>$ Stratiform $Q_{2}, Q_{2}^{\mathrm{s}}\left(\mathrm{K} /\right.$ day) $(\mathrm{F} 7.2)$ : Contribution to $Q_{2}$ from the "stratiform" columns.

19. $<\mathbf{1}>$ Radiative heating rate, $Q_{R}(\mathrm{~K} /$ day $)(\mathrm{F} 7.2)$

20. $<\mathbf{1}>$ Solar (short-wave) radiative heating rate, $Q_{R}^{\mathrm{SW}}(\mathrm{K} /$ day) $(\mathrm{F} 7.2)$

21. $<\mathbf{1}>$ Infrared (long-wave) radiative heating rate, $Q_{R}^{\mathrm{LW}}(\mathrm{K} /$ day) $(\mathrm{F} 7.2)$

22. $<\mathbf{1}>$ Clear radiative heating rate, $Q_{R}^{\text {clr }}(\mathrm{K} /$ day $)(\mathrm{F} 7.2)$ : The average radiative heating rate in the "clear" columns.

23. $<\mathbf{1}>$ Cloudy radiative heating rate, $Q_{R}^{\text {cld }}(\mathrm{K} /$ day $)(\mathrm{F} 7.2)$ : The average radiative heating rate in the "cloudy" columns.

24. $<2>$ Cloud mass flux, $M_{c}\left(\mathrm{mb} \mathrm{s}^{-1}\right)(\mathrm{F} 8.5): M_{c}=M_{u}-M_{d}$

25. $<2>$ Updraft cloud mass flux, $M_{u}\left(\mathrm{mb} \mathrm{s}^{-1}\right)(\mathrm{F} 7.5)$ :

$$
M_{u}=\frac{\sum_{j} \sigma \omega^{+}}{\sum_{j}}
$$

where $j$ is the grid point column index, $\sigma$ is the cloud fraction (defined previously), $\omega^{+}=\omega$ if $\omega<0$, otherwise, $\omega^{+}=0$. 
26. $<2>$ Downdraft cloud mass flux, $M_{d}\left(\mathrm{mb} \mathrm{s}^{-1}\right)(\mathrm{F} 7.5): M_{d}=M_{d s}+M_{d u}$, where $M_{d s}$ and $M_{d u}$ are the saturated and unsaturated downdraft cloud mass fluxes:

$$
M_{d s}=\frac{\sum_{j} \sigma \omega^{-}}{\sum_{j}}
$$

and

$$
M_{d u}=\frac{\sum_{j} \sigma_{p} \omega^{-}}{\sum_{j}}
$$

Here, $\omega^{-}=|\omega|$ if $\omega>0$, otherwise, $\omega^{-}=0$. Also, $\sigma_{p}=1$ if $\sigma=0$ and $q_{r}+q_{s}+q_{g}>P$, with $P=0.1 \mathrm{~g} / \mathrm{kg}$; otherwise, $\sigma_{p}=0$.

27. $<\mathbf{3}>$ Fractional area of updraft cores, $\overline{\sigma_{u}}$ (unitless) (F6.3): A "core" exists if $|\omega|>$ $W$, with $W=0.1 \mathrm{mb} / \mathrm{s}$. Thus,

$$
\overline{\sigma_{u}}=\frac{\sum_{j} \sigma_{u}}{\sum_{j}},
$$

where $\sigma_{u}=1$ if $\omega<-W$; otherwise, $\sigma_{u}=0$.

28. $<\mathbf{3}>$ Fractional area of downdraft cores, $\overline{\sigma_{d}}$ (unitless) $(\mathrm{F} 6.3)$ :

$$
\overline{\sigma_{d}}=\frac{\sum_{j} \sigma_{d}}{\sum_{j}}
$$

where $\sigma_{d}=1$ if $\omega>W$; otherwise, $\sigma_{d}=0$.

29. $<\mathbf{3}>$ Average core updraft speed, $\omega_{u}(\mathrm{mb} / \mathrm{s})(\mathrm{F} 7.3)$ :

$$
\omega_{u}=\frac{\sum_{j} \sigma_{u} \omega}{\sum_{j} \sigma_{u}} .
$$

30. $<\mathbf{3}>$ Average core downdraft speed, $\omega_{d}(\mathrm{mb} / \mathrm{s})(\mathrm{F} 7.3)$ :

$$
\omega_{d}=\frac{\sum_{j} \sigma_{d} \omega}{\sum_{j} \sigma_{d}} .
$$

\subsubsection{Time series}

Submit the results for each of the three groups of quantities listed below as a separate $A S C I I$ file as described in the subsection after the description of quantities to submit.

Group 1:

1. $<\mathbf{1}>$ Time of mid-point of averaging interval, $\bar{t}(\mathrm{~h})(\mathrm{F} 6.1)$

2. $<\mathbf{1}>$ Surface skin temperature, SST (K) (F7.2) 
3. $<\mathbf{3}>$ Near-surface dry static energy, $\overline{s_{0}}(\mathrm{~kJ} / \mathrm{kg})(\mathrm{F} 7.2): s=c_{p} T+g z$. "Near-surface" is the first model level above the surface.

4. $<\mathbf{3}>$ Near-surface water vapor mixing ratio, $\overline{q_{0}}(\mathrm{~g} / \mathrm{kg})(\mathrm{F} 6.2)$

5. $<\mathbf{3}>$ Near-surface moist static energy, $\overline{h_{0}}(\mathrm{~kJ} / \mathrm{kg})(\mathrm{F} 7.2): h=s+L q$.

6. $<\mathbf{3}>$ Near-surface horizontal wind velocity in x-direction, $\overline{u_{0}}(\mathrm{~m} / \mathrm{s})(\mathrm{F} 7.2)$

7. $<\mathbf{3}>$ Near-surface horizontal wind velocity in y-direction, $\overline{v_{0}}(\mathrm{~m} / \mathrm{s})(\mathrm{F} 7.2)$

8. $<\mathbf{1}>$ Surface turbulent flux of sensible heat, $\overline{\left(F_{s}\right)_{0}}\left(\mathrm{~W} / \mathrm{m}^{2}\right)(\mathrm{F} 6.1): F_{s} \equiv \bar{\rho} c_{p}\left(\frac{\bar{p}}{p_{0}}\right)^{R / c_{p}}\left\langle w^{\prime \prime} T^{\prime \prime}\right\rangle$.

9. $<\mathbf{1}>$ Surface turbulent flux of latent heat, $L \overline{\left(F_{q}\right)_{0}}\left(\mathrm{~W} / \mathrm{m}^{2}\right)(\mathrm{F} 6.1): F_{q} \equiv \bar{\rho}\left\langle w^{\prime \prime} q^{\prime \prime}\right\rangle$.

10. $<\mathbf{2}>$ Surface turbulent flux of horizontal momentum component in x-direction, $\overline{\left(F_{u}\right)_{0}}$ $\left(\mathrm{nt} / \mathrm{m}^{2}\right)(\mathrm{F} 8.4): F_{u} \equiv \bar{\rho}\left\langle u^{\prime \prime} w^{\prime \prime}\right\rangle$.

11. $<\mathbf{2}>$ Surface turbulent flux of horizontal momentum component in y-direction, $\overline{\left(F_{v}\right)_{0}}$ $\left(\mathrm{nt} / \mathrm{m}^{2}\right)(\mathrm{F} 8.4): F_{v} \equiv \bar{\rho}\left\langle v^{\prime \prime} w^{\prime \prime}\right\rangle$.

12. $<\mathbf{1}>$ Boundary layer depth, $\overline{Z_{i}}(\mathrm{~m})(\mathrm{F} 6.0)$

\section{Group 2:}

1. $<\mathbf{1}>$ Time of mid-point of averaging interval, $\bar{t}(\mathrm{~h})(\mathrm{F} 6.1)$

2. $<\mathbf{1}>$ Surface downwelling solar radiative flux, $\overline{\left(F_{S W}^{-}\right)_{0}}\left(\mathrm{~W} / \mathrm{m}^{2}\right)(\mathrm{F} 7.1)$

3. $<\mathbf{1}>$ Surface upwelling solar radiative flux, $\overline{\left(F_{S W}^{+}\right)_{0}}\left(\mathrm{~W} / \mathrm{m}^{2}\right)(\mathrm{F} 7.1)$

4. $<\mathbf{1}>$ Surface downwelling infrared radiative flux, $\overline{\left(F_{L W}^{-}\right)_{0}}\left(\mathrm{~W} / \mathrm{m}^{2}\right)(\mathrm{F} 6.1)$

5. $<\mathbf{1}>$ Surface upwelling infrared radiative flux, $\overline{\left(F_{L W}^{+}\right)_{0}}\left(\mathrm{~W} / \mathrm{m}^{2}\right)(\mathrm{F} 6.1)$

6. $<\mathbf{1}>$ TOA (top of atmosphere) downwelling solar radiative flux, $\overline{\left(F_{S W}^{-}\right)_{T}}\left(\mathrm{~W} / \mathrm{m}^{2}\right)$ (F7.1)

7. $<\mathbf{1}>$ TOA upwelling solar radiative flux, $\overline{\left(F_{S W}^{+}\right)_{T}}\left(\mathrm{~W} / \mathrm{m}^{2}\right)(\mathrm{F} 6.1)$

8. $<\mathbf{1}>$ TOA upwelling infrared radiative flux (OLR), $\overline{\left(F_{L W}^{+}\right)_{T}}\left(\mathrm{~W} / \mathrm{m}^{2}\right)(\mathrm{F} 6.1)$

9. $<\mathbf{1}>$ Cloud amount, $\overline{A_{\text {cld }}}$ (unitless) (F6.3): Fraction of columns which are "cloudy" for CRMs. For SCMs, this depends on cloud layer overlap assumptions.

10. $<\mathbf{2}>$ Cold cloud top area, $\overline{A_{\text {cld }}^{\text {cold }}}$ (unitless) $(\mathrm{F} 6.3)$ : Fraction of columns for which the "cloud top temperature" is less than $245 \mathrm{~K}$. 
11. $<\mathbf{1}>$ Precipitable water, $\overline{\mathrm{PW}}\left(\mathrm{kg} / \mathrm{m}^{2}\right)(\mathrm{F} 6.2): \mathrm{PW}=\int_{0}^{z_{T}} \bar{\rho} q d z$, where $z_{T}$ is the model top height.

12. $<\mathbf{1}>$ Cloud liquid water path, $\overline{\mathrm{LWP}}\left(\mathrm{kg} / \mathrm{m}^{2}\right)(\mathrm{E} 10.3): \mathrm{LWP}=\int_{0}^{z_{T}} \bar{\rho} q_{c} d z$.

13. $<\mathbf{1}>$ Cloud ice path, $\overline{\mathrm{IWP}}\left(\mathrm{kg} / \mathrm{m}^{2}\right)(\mathrm{E} 10.3): \operatorname{IWP}=\int_{0}^{z_{T}} \bar{\rho} q_{i} d z$.

\section{Group 3:}

1. $<\mathbf{1}>$ Time of mid-point of averaging interval, $\bar{t}(\mathrm{~h})(\mathrm{F} 6.1)$

2. $<\mathbf{3}>$ Vertically integrated rain, $\overline{\mathrm{RP}}\left(\mathrm{kg} / \mathrm{m}^{2}\right)(\mathrm{E} 10.3): \mathrm{RP}=\int_{0}^{z_{T}} \bar{\rho} q_{r} d z$.

3. $<\mathbf{3}>$ Vertically integrated snow, $\overline{\mathrm{SP}}\left(\mathrm{kg} / \mathrm{m}^{2}\right)(\mathrm{E} 10.3): \mathrm{SP}=\int_{0}^{z_{T}} \bar{\rho} q_{s} d z$.

4. $<\mathbf{3}>$ Vertically integrated graupel, $\overline{\mathrm{GP}}\left(\mathrm{kg} / \mathrm{m}^{2}\right)(\mathrm{E} 10.3): \mathrm{GP}=\int_{0}^{z_{T}} \bar{\rho} q_{g} d z$.

5. $<\mathbf{1}>$ Surface rainfall rate, $\bar{P}(\mathrm{~mm} /$ day $)(\mathrm{F} 7.2)$ : Calculate from the surface rain accumulated over every time step.

6. $<\mathbf{2}>$ Convective surface rainfall rate, $\overline{P_{c}}(\mathrm{~mm} /$ day $)(\mathrm{F} 7.2)$ : The contribution to $\bar{P}$ from the "convective" columns.

7. $<\mathbf{2}>$ Stratiform surface rainfall rate, $\overline{P_{s}}(\mathrm{~mm} /$ day $)($ F7.2): The contribution to $\bar{P}$ from the "stratiform" columns.

8. $<\mathbf{3}>$ Rain fractional area, $\overline{A_{r}}$ (unitless) $(\mathrm{F} 6.3)$ : Fraction of columns which are "rainy."

9. $<\mathbf{1}>$ Convective fractional area, $\overline{A_{c}}$ (unitless) (F6.3): Fraction of columns which are "convective."

10. $<\mathbf{1}>$ Stratiform fractional area, $\overline{A_{s}}$ (unitless) (F6.3): Fraction of columns which are "stratiform."

\subsection{Format for Submitted files}

Naming conventions are given at the end of subsection 2.2.2.

\subsubsection{Format for profile quantities}

Submit the results for each quantity listed above as a separate ASCII file in the file format described in this subsection. Include the time at the midpoint of the averaging interval $\bar{t}$ (hours, F6.1) and either (a) the pressure $p(\mathrm{mb}, \mathrm{F} 7.3)$ or (b) the height $z(\mathrm{~km}, \mathrm{~F} 7.3)$ as the $x$ and $y$ coordinates, $x$ moving fastest.

The file structure is: 


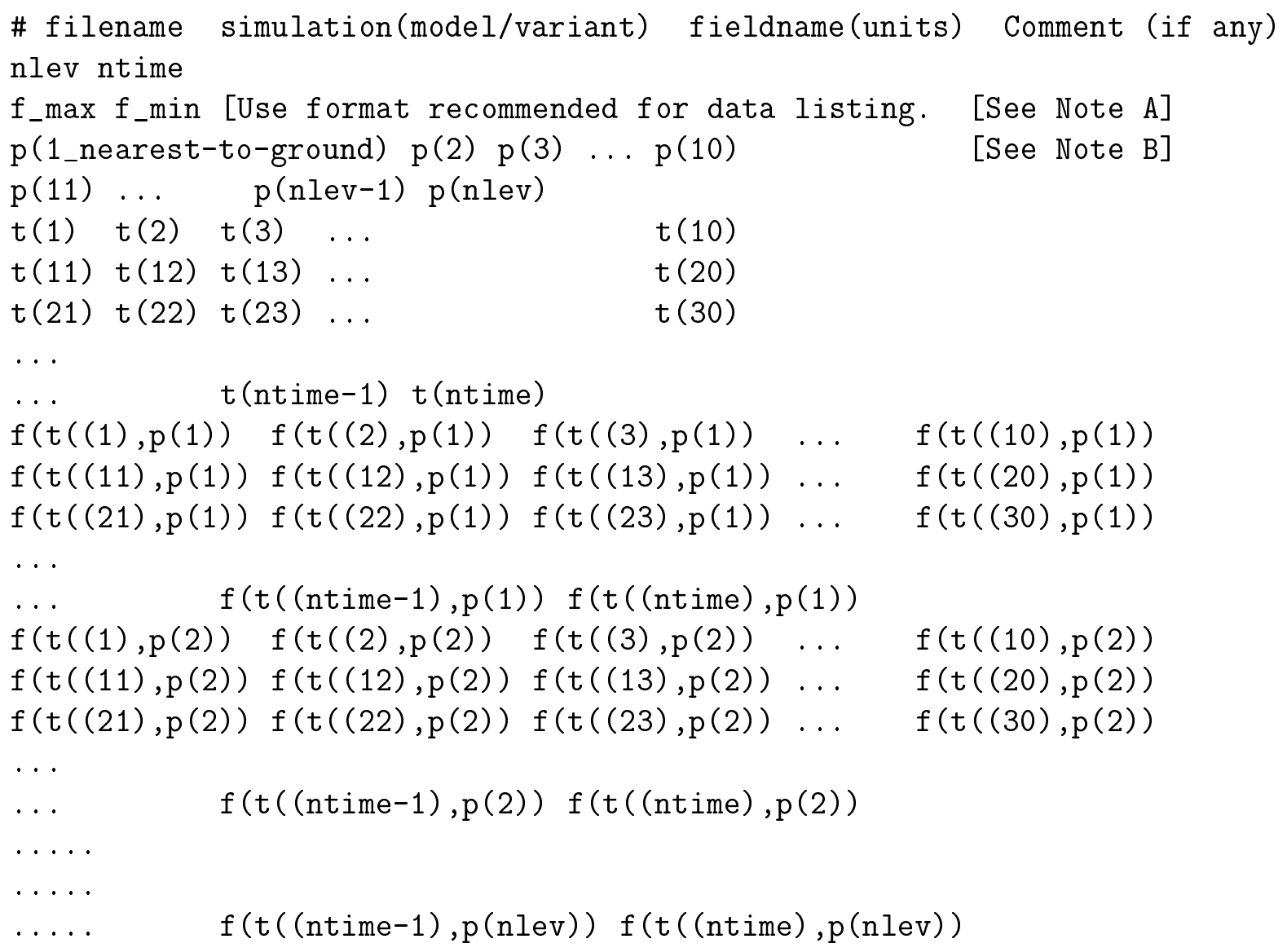

Note $A$ - IMPORTANT - For each field, the max and min values should be written here, using the same data format as recommended for writing all data values of that field. For each simulation, and each field - check to be sure that the max/min values would not and did not overflow the field format you chose to use. If either max or min did, it and other values (lesser but of same magnitude) would be unreadable, as they might have been printed as $* * * *$ or be taking up the delimiting blank space between the values. Excessive min or max values could indicate calculation or unit errors on the part of your model or its output code. If you are sure your code is O.K., feel free to increase the format length, to accommodate the values of magnitude larger than expected. The important thing is that the values are printed with a delimiting blank space and, needless to say, with valid characters.

Note $B$ - For models that do not have isobaric levels, the block for level values can be those of height or sigma. In these cases, each simulation run should have a .p0. file, which contains the pressure data values that vary with time as well as with height. Also, .p1. file should contain height data, if heights vary over time. The .p1. file need not be written, if height values are constant over time and are listed in each file as coordiate data for the levels.

Note $C$ - If you don't plan on submitting a certain field, please submit an empty file with the prescribed name and SUFFIXED '.not_submitted'. The presence of this dummy 
file saves recipients of your data set from wondering about whether the data files were otherwise misplaced or not, and also saves yourself from the bother of having to answer such queries. Please be sure submit a dummy file, especially if it is a required field for which you do not intend to submit data.

\subsubsection{Format for time series quantities}

Submit the results for each of the three groups of quantities listed above as a separate ASCII file. Name each file as follows: simulation.tgroup.modeler. For example, the file name "a.t1.krueger" indicates simulation $=\mathrm{a}$, time series group $=1$, and modeler $=$ krueger.

In the first line of each file, please include a comment identifier (\#), the filename, and, optionally, any further distinguishing characteristics. Follow this with the results; use one line for each time.

The structure of the file is:

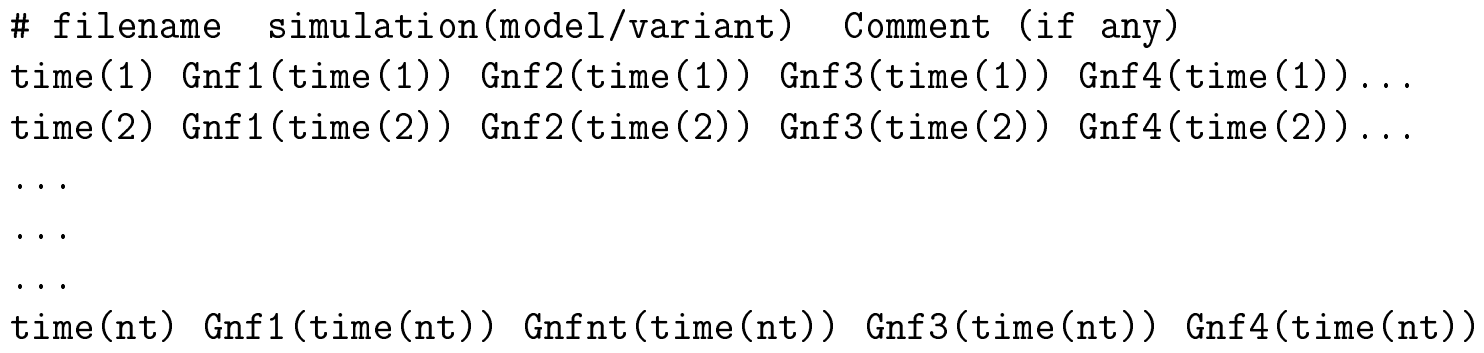

Note $A$ - For time series data files (.t1, .t2, .t3), the format for each data line does not allow for extra delimiting space between any adjacent pair of the time field and/or the data fields, besides the positions in the formats left unused by the data values.

Note $B$ - If you don't plan on submitting a certain field, please write in its format space a negative number with all decimal positions being ' 8 ' except the exponent positions, which can be just ' +00 ' or ' +01 '. If you do not intend to submit any field of a certain file, you may simply submit an empty file with an appropriate name, ans SUFFIXED '.not_submitted', to save recipients from wondering.

Suggested formats for each quantity are included in the descriptions below using Fortran notation. So that each line includes no more than 80 characters (necessary for e-mail transmission), simply write each line using the suggested formats. For example, the format for each line for group 1 is (F6.1,2F7.2,F6.2,3F7.2,2F6.1, 2F8.4). For group 2, it is (F6.1,2F7.1,2F6.1,F7.1,2F6.1,2F6.3,F6.2,2E10.3), and for group 3 it is (F6.1,3E10.3,3F7.2,3F6.3).

If the files are transmitted via ftp, all that is required is that the results for each time are contained within one record (a line ending with a carriage return) of any length, and that each quantity (including the first) be preceded by at least one space. The format can be fixed point or E- (exponential) format. 


\section{Model Description}

In order to compare results from different models, workshop participants are asked to complete the following description relevant for simulations performed for the intercomparison. This description will help to build up the workshop report at the final stage. Note: the following items apply to both SCMs and CRMs. Respond only to those that apply to your SCM.

- Scientist name

Affiliation

Address

Email address

Fax Number

- Model type (1D, 2D, 3D)

- Numerical Domain

Domain size in $\mathrm{x}$-direction:

Domain size in $\mathrm{y}$-direction:

Domain size in z-direction:

Number of grid points in $\mathrm{x}$-direction:

Number of grid points in $\mathrm{y}$-direction:

Number of grid points in z-direction:

Grid size in x-direction:

Grid size in y-direction:

Grid size in z-direction (if stretched please specify):

- Time step

- Numerical Technique

Numerical method (finite-difference, spectral, etc.):

Advection scheme and its order of accuracy:

Time scheme and its order of accuracy:

Dynamical equations (elastic, anelastic, etc.):

Numerical diffusion (type, order, magnitude of coefficient) 
Upper boundary condition (Sponge layer, specification, ...):

Other information

- Physical Parameterizations:

Surface flux parameterization for heat, moisture, momentum:

Longwave radiation parameterization:

Shortwave radiation parameterization:

How were radiative fluxes above the computational domain handled?

Microphysical (2D/3D models) or cloud/convective (1D model) parameterization: type, number of hydrometeor classes, ...

Turbulence closure scheme (turbulence closure type, variables predicted and diagnosed by the turbulence closure, closure for turbulent length scale, ...)

Other information

- Documentation: Please provide references that more fully describe your model, if available. 